\title{
Fast magnetic reconnection and the coalescence instability
}

\author{
G. J. Rickard and I. J. D. Craig \\ University of Waikato, Hamilton, New Zealand
}

(Received 29 June 1992; accepted 25 September 1992)

\begin{abstract}
The magnetic reconnection that occurs during the nonlinear development of the coalescence instability is considered. The structure of the reconnection region at the time of maximum current as a function of the resistivity $\eta$ is analyzed in detail using a compressible magnetohydrodynamic fluid code. It is shown that the numerical results concur remarkably well with a simple scaling analysis which predicts the dependence of the reconnection region structure on $\eta$. It is argued that the flow topology is crucial in maintaining the "fast" reconnection rate. The results indicate a flux pileup solution in which the flux annihilation rate is approximately independent of $\eta$, whereas the Ohmic dissipation rate scales as $\eta^{-1 / 3}$. The possibility that these scalings break down at lower values of $\eta$ is discussed.
\end{abstract}

\section{INTRODUCTION}

Magnetic reconnection is recognized as a key physical ingredient in the evolution of astrophysical and terrestrial plasmas. Reconnection allows topological simplification of the magnetic field by converting "topologically bound" magnetic energy into plasma heating and the kinetic energy of mass motion.

The magnetic Reynolds number is an important parameter in magnetic reconnection theory: $R_{m}=L V / \eta$, where $\eta$ is the plasma resistivity and $L$ and $V$ are characteristic lengths and velocities, respectively. The small resistivity in astrophysical plasmas means that $R_{m}$ is generally very large, implying large reconnection time scales (over several days) in most applications. In a solar flare, however, the collapse of the coronal magnetic field occurs explosively-over $100 \mathrm{sec}$ or so. This implies that the reconnection rate can depend only weakly on $R_{m}$ if reconnection is to be viable as a flare release mechanism. The determination of the scaling of the reconnection rate with resistivity therefore lies at the heart of solar flare theory. Is the reconnection "fast"-i.e., independent of any positive power of $\eta$ ? It is this problem that we explore here.

Although reconnection is a fundamental process, configurations that result in "spontaneous" reconnection have been comparatively little studied. In the past much effort has been focused on the steady-state merging of externally driven antiparallel magnetic fields as typified by the Sweet-Parker mechanism (Sweet ${ }^{1}$ and Parker $^{2}$ ) and the Petschek ${ }^{3}$ developments (e.g., see the reviews of Vasyliunas ${ }^{4}$ and Forbes and Priest ${ }^{5}$ ). Only comparatively recently have dynamically complete simulations been investigated. Yet the notion that reconnection could occur as a natural outgrowth of some ideal magnetohydrodynamic (MHD) instability has long been considered theoretically attractive. This view is supported by the buildup of localized currents in recent nonlinear, three-dimensional (3-D) simulations of the corkscrew kink instability (Craig and Sneyd ${ }^{6}$ ). In reality, of course, the nonresistive collapse toward a final configuration involving current singularities is always arrested by resistive diffusion. Reconnection then occurs naturally, uncompromized by the ad hoc specification of external factors that, in the steady-state approach, determine the driving of the system.
There is in fact one MHD process-the coalescence instability-that provides a simple two-dimensional (2-D) prototype for spontaneous reconnection (see Finn and $\mathrm{Kaw}^{7}$ and the numerical simulations of Pritchett and $\mathrm{Wu}^{8}$ ). The equilibrium consists of a chain of magnetic islands. These are subject to an ideal instability in which pairs of neighboring islands sharing a common $X$-type neutral point begin to pile up flux at the $X$ point. In the absence of resistivity the fiux in each island pilcs up indefinitely forming a singularity. Finite resistivity, however, allows the flux to be annihilated so that adjacent magnetic islands can merge together and coalesce, effectively liberating the energy associated with the ideal singularity.

The self-consistent nature of the coalescence instability has already attracted the reconnection study of Biskamp and Welter $^{9}$ (hereafter BW). In a remarkable investigation BW empirically deduce the scalings with resistivity of certain key parameters describing the reconnection region. Some of these scalings are surprising-for instance the Ohmic dissipation rate at the sheet increases as the resistivity declines!-and to our knowledge they have not been confirmed (or denied) by subsequent workers. DeLuca and $\mathrm{Craig}^{10}$ (hereafter DC) demonstrate fast incompressible reconnection (in two and one-half dimensions) for a disturbed equilibrium field in periodic geometry, while Strauss, ${ }^{11}$ working in an entirely different topology, is "surprised" by a BW type scaling for the width of the current layer. However, while these studies provide some evidence for fast reconnection, the ultrafast BW scalings remain unexplained.

The aim of the present paper is twofold: first, to present a simple theoretical framework for the interpretation of dynamic reconnection simulations; second, to present a detailed computational investigation of the compressible reconnection that occurs via the development of the coalescence instability. As motivation we note that while the phenomenological features of the coalescence instability have been tackled by previous workers (e.g., Bhattacharjee et al..$^{12}$ and Tajima and Sakai ${ }^{13}$ ), the crucial dependence of the reconnection rate with resistivity has not been explored since $\mathrm{BW}$, either in terms of theoretical scaling arguments or detailed computational experiments.

The outline of the paper is as follows. Section II details 
the derivation of the theoretical scaling laws. In particular, the BW scaling is determined within the theory. In Sec. III we describe the equilibrium configuration, the model equations, and the numerical method of solution. The results are presented in Sec. IV, and in Sec. V we finish with conclusions regarding our results and a discussion of their implication for magnetic reconnection.

\section{THEORETICAL SCALING LAWS}

The engine room of flux reconnection and magnetic energy dissipation is the current sheet, for it is the scaling of the sheet parameters with resistivity that controls the speed of magnetic annihilation. In fact, there are two potential measures of the magnetic annihilation rate: one is the rate of flux annihilation at the neutral point; the other is the rate of Ohmic dissipation in the sheet. These determine in what form the magnetic energy is released, whether as the kinetic energy of mass motion, or as in situ Joule heating of the plasma. We shall follow DC and call the release of the magnetic energy "fast" if either of these measures scale independently of any positive power of $\eta$. In coronal plasmas $\eta \sim 10^{-12}$ so the energy release has to be fast to account for the explosive time scale of the solar flare.

The complexity of the dynamic plasma-field coupling generally precludes any closed-form solution for the current sheet structure. In what follows we derive simple scaling laws in an attempt to quantify the essential dependencies of the reconnection problem. We consider a twodimensional system in which the magnetic field is described by a planar flux function via $B=\nabla \psi \times \hat{z}$. The system contains a current sheet of length $L$ and width $l$ that straddles a neutral point at the origin and forms a stagnation point of the flow.

\section{A. Steady-state scaling laws}

In the classical steady-state picture material flows into the sheet with speed $u$, and flows out with speed $v$. Conservation of mass gives,

$$
\rho_{\mathrm{e}} u L=\rho_{i} u l,
$$

where $\rho_{e}$ and $\rho_{i}$ are the external and internal densities with respect to the sheet.

The advection of flux into diffusion region is balanced by the diffusion of flux through the sheet, giving

$$
u=\eta / l \text {. }
$$

This criterion effectively locates the edge of the current sheet: inside the resistive layer the problem is diffusion dominated while outside the problem is advection dominated. In the Sweet-Parker model the gas is incompressible, the external field is presumed uniform and the current sheet length is fixed by the geometry. Simple considerations of momentum balance along the sheet then show that the exhaust velocity $v$ can be identified with the Alfvén speed of the external field: the current sheet effectively squeezes out the exhaust material. It follows that $v$, in common with the external field $\mathbf{B}$ and $L$, is invariant with $\eta$. Equations (1) and (2), along with Ampère's law $J=B /$ $l$ for the sheet, are sufficient to determine the scalings of the Sweet-Parker mode. In particular, the flux annihilation rate at the neutral point $\partial \psi / \partial t=\eta J$ and the Ohmic dissipation rate $W_{\eta}=\eta J^{2} l L$ scale as

$$
\frac{\partial \psi}{\partial t} \sim W_{\eta} \sim \eta^{1 / 2}
$$

This qualifies the Sweet-Parker mechanism as "slow."

The fast steady-state mechanism of Petschek can be viewed in much the same general terms as the SweetParker model. Petschek demonstrates that an external flow topology can be matched to a sheet of fixed aspect ratio in which both the length and width scale directly as $\eta$. The dissipation scalings are then

$$
\frac{\partial \psi}{\partial t} \sim \eta^{0}, \quad W_{\eta} \sim \eta^{1},
$$

with $u, v$, and the external $B$ invariant with $\eta$. The mechanism is fast but, because flux annihilation dominates Ohmic energy dissipation in the limit of small $\eta$, the bulk of the released energy emerges via a magnetic slingshot as the kinetic energy of mass motion. The Petschek model has many defects-- the model is known to be nonunique, for example-but it is important historically as the first demonstration of fast reconnection. Dynamic reconnection studies, however, have provided little support for the Petschek mechanism (e.g., Biskamp; ${ }^{14}$ cf. Forbes and Priest).

\section{B. Dynamic scaling laws}

At first sight it might appear that the steady-state relations used previously are inapplicable in a fully dynamic situation. However, the linear theory of dynamic reconnection developed by Craig and McClymont ${ }^{15,16}$ and Craig and Watson ${ }^{17}$ suggests that the continuity and boundarylayer relations can be applied throughout the development of the current layer in conjunction with a further relation that determines the excess magnetic energy in the sheet. The signature of fast reconnection is then provided by the scaling of the current layer at the time of maximum current.

We first note that a central assumption of the steadystate model - that the magnetic field at the onset of the current layer is invariant with $\eta$-cannot be true dynamically. We need only consider the superposition of a topological perturbation on an equilibrium $\mathrm{X}$-point configuration under the assumption that magnetic forces are dominant (Craig and Watson). A magnetic wave develops that localizes close to the neutral point driving massive currents which become unbounded in the absence of resistive diffusion. An invariant magnetic energy is associated with the formation of the current layer, i.e.,

$$
B^{2} L l=\text { invariant, }
$$

where $B$ now represents the magnetic field at the onset of the sheet. The magnetic intensity continues to build up until resistive effects come into play.

The linear theory also implies that $L$ is invariant with $\eta: L$ is determined by the canceling of the equilibrium field 
by the perturbation field and, within limits imposed by the geometry, depends only on the properties of the initial disturbance (see Craig and Watson). This explains an almost universal feature of dynamic reconnection experiments, namely, the observed constancy of the current sheet length with $\eta$ (see Biskamp). The width of the current layer, however, depends quite critically on the resistivity-it is a crucial factor, along with the magnetic intensity at the sheet, in determining the rate of the magnetic annihilation.

Let us combine Eqs. (1), (2), and (5) for the case of an incompressible plasma. Remembering that the exhaust speed is determined by the Alfvén speed associated with $B$ at the onset of the sheet, we find that

$$
\frac{\partial \psi}{\partial t} \sim \eta^{0}, \quad W_{\eta} \sim \eta^{-1 / 3},
$$

with $u \sim \eta^{1 / 3}, v \sim B \sim \eta^{-1 / 3}, l \sim \eta^{2 / 3}$. These are exactly the scalings determined numerically by $\mathrm{BW}$ for coalescencedriven reconnection. This means that the flux pileup predicted by Eq. (5) must hold good for nonlinear reconnection at least over the modest (two decade) range of resistivities considered. A similar "ultrafast" scaling has also been observed by Strauss.

There are, however, other possible scalings for fast reconnection. Suppose we assume an incompressible flow topology consistent with stagnation point flow. In this case the exhaust flow pattern is determined by the global length scale $L$ rather than the size scale $l$ of the diffusion region: $v$ is then invariant but the outflow is no longer restricted to a narrow wedge of angle $l / L$ as in the Sweet-Parker and Petschek models. The previous arguments then predict the flux pileup scaling obtaincd empirically by $D C$, namely,

$$
\frac{\partial \psi}{\partial t} \sim \eta^{1 / 4}, \quad w_{\eta} \sim \eta^{0},
$$

with $u \sim \eta^{1 / 2}, B \sim \eta^{-1 / 4}, l \sim \eta^{1 / 2}$. This scaling, though not as spectacular as the $\mathrm{BW}$ result, remains fast with the bulk of the magnetic energy liberated as heat rather than the kinetic energy of mass motion.

The question of what determines the reconnection rate in practice thus seems closely bound up with the nature of the flow topology. This point has already been emphasized by Forbes and Priest in the context of steady-state reconnection models. In the case of dynamic reconnection, it is known from linear theory (Craig and Watson) that fast reconnection in a simple closed $X$ point is stalled by the inclusion of gas pressure. This means that the geometry cannot be too simple if fast reconnection is to persist in the extreme case of an incompressible plasma: the flow topology must contain sufficient freedom to allow the buildup of current in the diffusion layer to remain unimpeded by gas pressure effects. The two-dimensional circular flow patterns obtained by BW and DC apparently satify this requirement, at least for simulations over a modest range of plasma resistivities.

Finally we note that in deriving the previous scalings we have considered only the simplest case of an incompressible plasma, i.e., $\rho_{e}=\rho_{i}$ in Eq. (1). Compressibility provides extra degrees of freedom that cannot be elimi- nated on the basis of the previous scaling arguments. However, because the incompressible limit reflects the maximal negative feedback of the gas on the system, we would not expect finite compressibility to slow the reconnection rate. One aim of the present computational simulations is to see which scalings persist in the presence of significant compressibility effects.

\section{FORMULATION OF THE PROBLEM}

We first discuss the initial conditions that determine the ideal coalescence instability. In fact we study two classes of equilibria: one class is purely two dimensional with no perpendicular component to the planar field; the second defines force-free field solutions for nonvanishing $B_{z^{*}}$ We go on to describe the evolution equations and their computational implementation.

\section{A. The initial conditions}

In a two-dimensional system with an ignorable $z$ coordinate we write the magnetic field $\mathbf{B}(x, y)$ as,

$$
\mathbf{B}=\mathbf{B}_{\perp}+B_{z} \hat{\mathbf{z}}=\nabla \psi \times \hat{\mathbf{z}}+B_{z} \hat{\mathbf{z}},
$$

with the planar field components given by

$$
B_{x}=\frac{\partial \psi}{\partial y} \text { and } B_{y}=-\frac{\partial \psi}{\partial x} .
$$

The force balance condition gives the equilibrium equation

$$
\nabla^{2} \psi+f(\psi)=0
$$

where

$$
\frac{d}{d \psi}\left(P+\frac{B_{z}^{2}}{2}\right)=f(\psi)
$$

The ideal MHD equilibrium we use is that representing a periodic chain of magnetic islands given by Fadeev et al. ${ }^{18}$ In this case

$$
\psi=-\frac{B_{0}}{k} \ln [\cosh (k y)+\epsilon \cos (k x)],
$$

which corresponds to the choice

$$
f(\psi)=J_{z}(x, y)=B_{0} k\left(1-\epsilon^{2}\right) \exp \left(\frac{2 k \psi}{B_{0}}\right) .
$$

Here $k$ and $B_{0}$ are normalization constants and $0 \leqslant \epsilon \leqslant 1$.

From Eq. (10) we see that there are two classes of equilibria. The first is purely two dimensional with $B_{z}=0$ and

$$
P=P_{0}+\frac{B_{0}}{k} \frac{f(\psi)}{2} .
$$

The second is force free with the gas pressurc a constant $P_{0 z}$, say, and

$$
\frac{B_{z}^{2}}{2}=\frac{B_{0 z}^{2}}{2}+\frac{B_{0}}{k} \frac{f(\psi)}{2} .
$$

The reconnective results of BW apply only to fields of the form given by Eq. (13) but here we consider both classes of equilibria. Following Pritchett and $\mathrm{Wu}$, we choose $k^{-1}$ to be the unit length. For $\epsilon=0.3, k^{-1}$ is approximately the island half-width. 


\section{B. The evolution equations and computational method}

Our simulations of the coalescence instability are governed by the resistive MHD equations,

$$
\begin{aligned}
& \frac{\partial \rho}{\partial t}+\nabla \cdot(\rho \mathbf{v})=0, \\
& \rho\left(\frac{\partial \mathbf{v}}{\partial t}+\mathbf{v} \cdot \nabla \mathbf{v}\right)=(\nabla \times \mathbf{B}) \times \mathbf{B}-\nabla P-v \mathbf{V},
\end{aligned}
$$

and

$$
\frac{\partial \mathbf{B}}{\partial t}=\nabla \times(\mathbf{v} \times \mathbf{B})+\eta \nabla^{2} \mathbf{B},
$$

where $\rho, \mathbf{v}, P$, and $\mathbf{B}$ are the normalized fluid density, velocity, pressure, and magnetic field, respectively; $V$ represents the viscous damping term. We express $\mathrm{v}=(u, v, w)$ in component form. For simplicity we use the isothermal relation $P \propto \rho$ as our equation of state.

For the equilibria with $B_{z}=0$ [Eq. (13)] the problem is purely two dimensional. However, for the force-free equilibria [Eq. (14)] the momentum equation [Eq. (16)] admits a flow in the $\hat{\mathbf{z}}$ direction $w(x, y)$, and $B_{z}(x, y)$ evolves in time through Eq. (17). The system is then said to be two and one half dimensional (two space, and three velocities).

As a first step, Eqs. (15) and (16) are integrated forward in time using a vectoral Lax-Wendroff formulation of the fluid equations (Craig and Watson). Theoretically, the full conservation properties of the system may be exploited by writing the Lorentz force as the divergence of the magnetic stress tensor; however, this course does not lead to a stable computational procedure. Thus we include the Lorentz term only as an explicit driving force in the vectoral fluid system, along with the viscous terms. The nonlinear overshoot of the Lax-Wendroff procedure is corrected in the usual manner, either by the addition of pseudoviscosity or by the inclusion of a flux limiter (Zalasek ${ }^{19}$ ).

The next step is to determine the evolution of the field. Using the flux function formulation of Eq. (8), Eq. (17) reduces to

$$
\frac{\partial \psi}{\partial t}+\mathbf{v} \cdot \nabla \psi-\eta \nabla^{2} \psi=0
$$

and

$$
\frac{\partial B_{z}}{\partial t}+\mathbf{y} \cdot \nabla B_{z}-\eta \nabla^{2} B_{z}=\nabla \cdot\left(w \mathbf{B}_{1}\right)-B_{z} \nabla \cdot \mathbf{v}
$$

This pair of equations is treated as a vectoral system using the alternating direction implicit formulation of Craig and Sneyd. ${ }^{20}$ The right-hand side of Eq. (18b) is handled as an explicit source term, but the velocities are correctly weighted using the updated values. The implicit weighting of the scheme is determined by the parameter $\theta$, where $0<\theta<1$ : for $\theta=0$ the scheme is explicit; for $\theta=1$ fully implicit. In general, for optimum stability and accuracy, we take $\theta=0.5$ : the system is then limited only by the advective time-step condition [i.e., the CourantFrederichs-Levy (CFL) restriction]. Having advanced Eqs. (18a) and (18b), the cycle for solving the full set of resistive MHD equations is complete.

\section{RESULTS}

As first discussed by Finn and Kaw and Pritchett and $\mathrm{Wu}$, the equilibrium given by $\mathrm{Eq}$. (11) is unstable to ideal instabilities for all values of $\epsilon$. Specifically, it is found that the instability growth rate is proportional to the equilibrium current as parametrized by $\epsilon$ in Eq. (12). We determine the unstable linear eigenfunctions with which to perturb the equilibrium velocity profile using techniques developed by Craig et al. ${ }^{21}$ This is not critical in driving the coalescence-indeed, a random perturbation will eventually produce the instability. However, as found by Pritchett and $\mathrm{Wu}$, simulation times can be reduced by having the initial perturbation as close to the true eigenfunction as possible. Since it can take of the order of 100-200 Alfvén transit times to reach peak current-depending on the compressibility and the $\eta$ value-using the true eigenfunctions helps to ease the computational burden.

We take the system to be periodic in $x$ and bounded by perfectly conducting walls in $y$. We exploit the symmetry of the system by modeling only one quadrant (as BW) on a uniform plane mesh, with $0 \leqslant x \leqslant x_{m}$ and $0 \leqslant y \leqslant y_{m}$. In our dimensionless units $x_{m}=2 \pi$ and $y_{m}$ is chosen large enough so that the presence of the bounding wall has only a small impact on the dynamics. Typically, $y_{m}=\pi$. For most runs 120 grid points in the $x$ direction, and 60 in the $y$ direction suffices. At smaller values of $\eta$, however, the resolution has to be at least doubled to model the current layer adequately.

We find that the extreme scaling of coalescence-driven reconnection places stringent demands on our computing rcsources. This is consistent with BW who quote a mesh spacing of $\Delta x=6 \times 10^{-3}$ for the current sheet for $\eta \leqslant 2 \times 10^{-4}$. For this reason we have chosen not to explore the dependence of the reconnection on $\epsilon$. Instead we fix $\epsilon=0.3$ and proceed to vary $\eta$.

We simulate not only the purely two-dimensional equilibrium used by BW [given by Eq. (13)], but also the force-free equilibrium given by Eq. (14). In both cases we always have $B_{0}=1$. For the Eq. (13) equilibrium, we have used $P_{0}=0.1$ and 1 to test compressibility effects. For the Eq. (14) equilibrium, $B_{0 z}=0$ and $P_{0 z}=0.5,1$, and 1.5. By varying the background gas pressure through $P_{0}$ or $P_{0 z}$, we are effectively changing the mass that has to be driven by the instability-hence the larger $P_{0}$ or $P_{0 z}$, the more "incompressible" an equilibrium is considered. Note that unlike Bhattacharjee et al., or Tajima and Sakai, we have not investigated the effects of varying $B_{0}$ or $B_{0 z}-$ we aim to emphasize the $\eta$ scalings. We shall therefore refer to either $P_{0}$ or $P_{0 z}$ to label a particular equilibrium. As always, the range of our parameters is determined by the resolution of the current sheet width at the smallest values of $\eta$.

\section{A. Morphology of the solutions}

Figure 1(a) shows the equilibrium magnetic structure. The peak of the toroidal current associated with an island lies at $x=x_{m} / 2, y=0$. It is the attraction between neighboring current channels that drives the coalescence. The form of the dominant unstable eigenmode is shown in Fig. 1(b). Following the linear phase, the instability drives the 
(a)

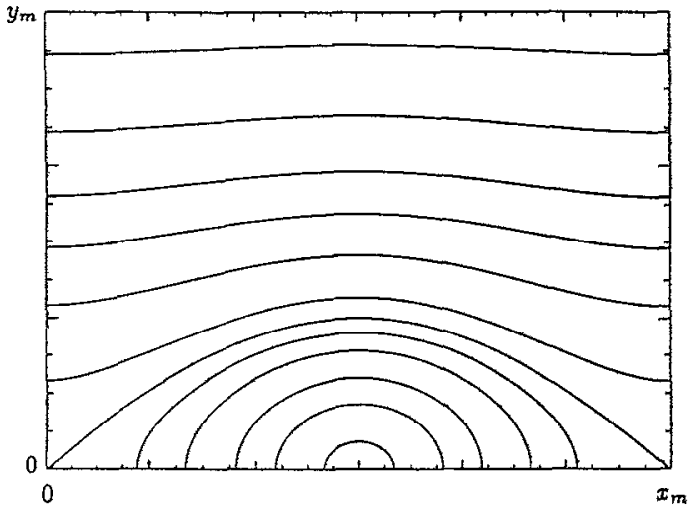

(b)

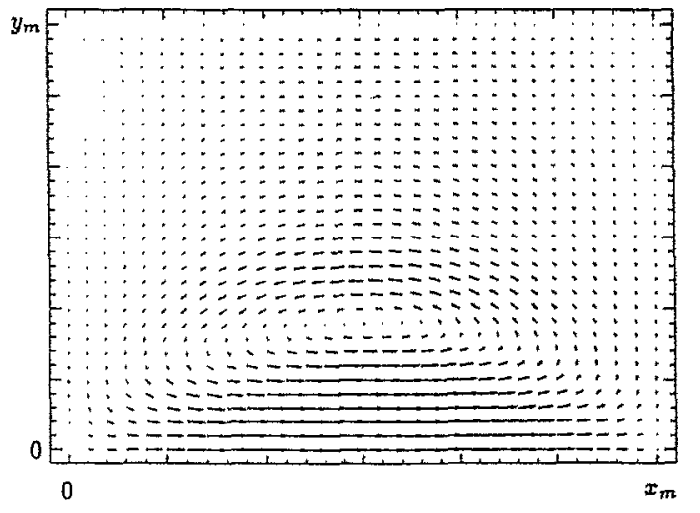

(c)

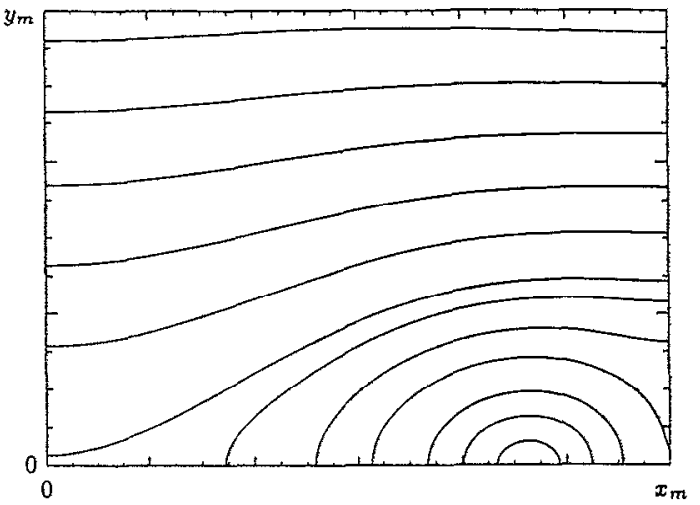

(d)

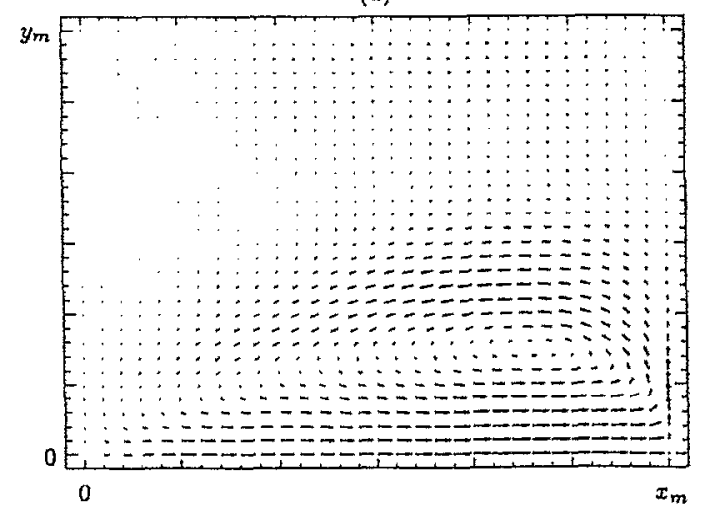

(e)

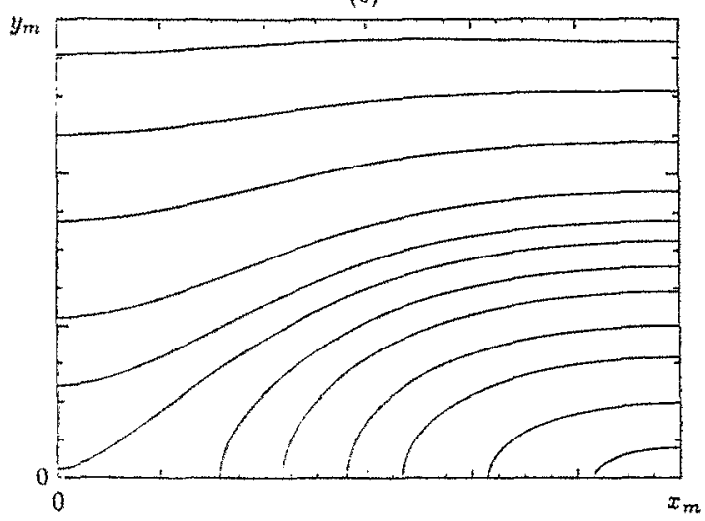

(b)

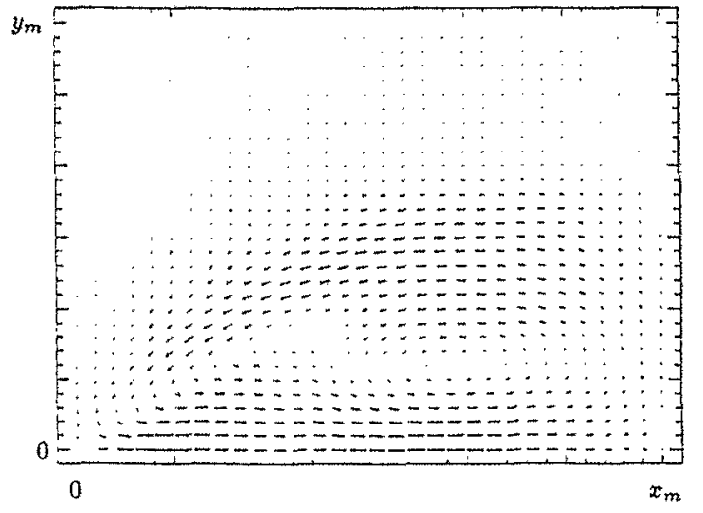

FIG. 1. Magnetic flux function contours and fluid flow vectors for the case $P_{0}=0.1$ and $\eta=5 \times 10^{-3}$. (a), (c), and (e) show contours of the magnetic flux function for the initial equilibrium (time $t=0$ ), for the time of peak current in the current sheet $(t=55.54)$, and well into the relaxation phase $(t=120.0)$, respectively, (b) shows the form of the unstable eigenfunction. (d) and ( $f$ ) show the fluid flow vectors coincident with the contours in (c) and (e). The maximum flow speed in (d) is 0.1093 , and in (f) is 0.0245. The flow speed is in units of the Alfven speed in the asymptotic magnetic field, and time is with respect to the Alfvern transit time across a unit length. 

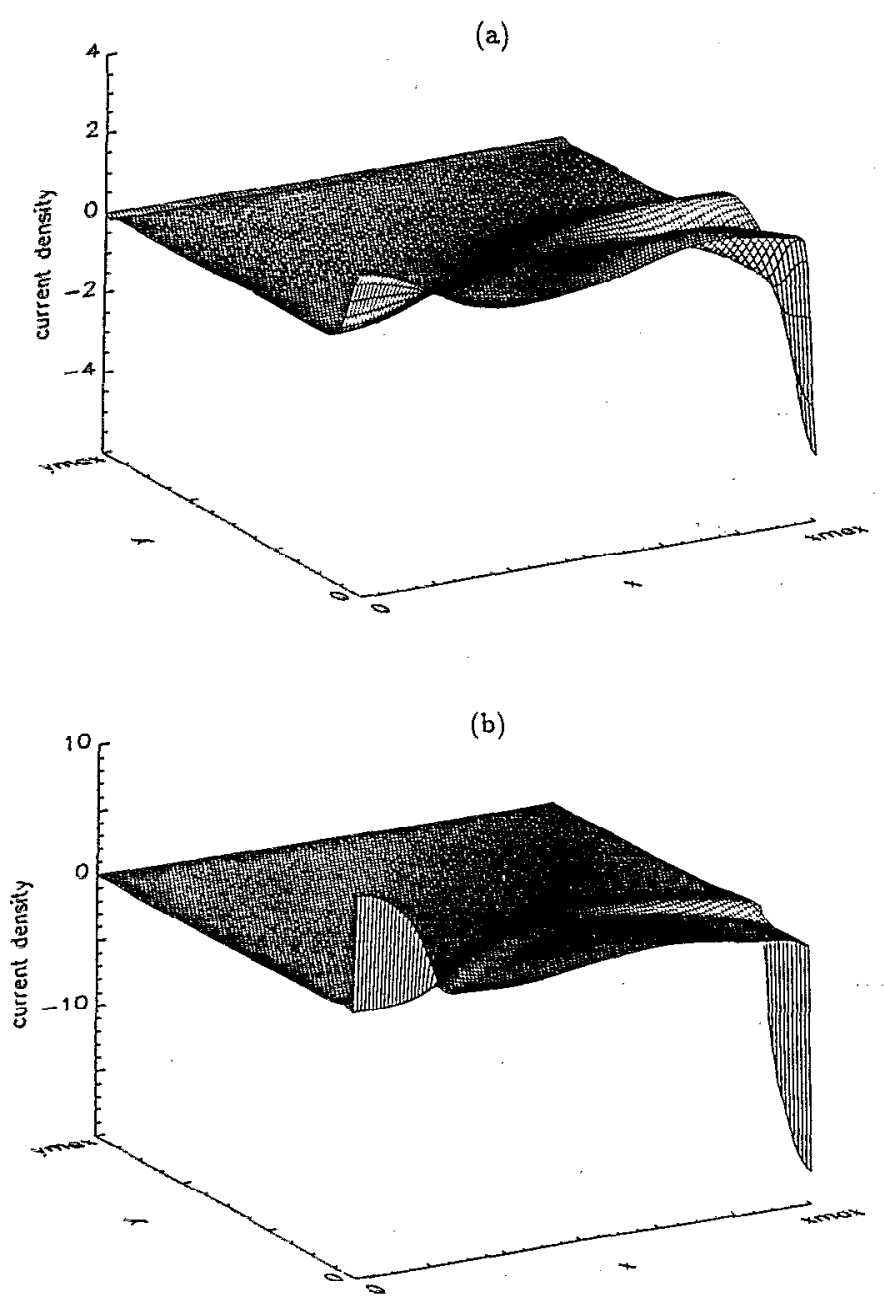

FIG. 2. Surface plots of the current density. (a) is at the time of peak current in the current sheet for the equilibrium of Fig. 1. The singular structures obtaincd with $\eta=0$ and artificial damping to remove the fluid energy are shown in (b).

field toward a singular configuration involving current sheets. The resultant flux surfaces can be seen in Fig. 1(c) which shows the right translation of the current channel, and the development of a current sheet at $x=x_{m}$. Figure 1 (c) coincides with the peak current in the current sheet. The flow topology at this time is shown in Fig. 1(d). The circulation of the linear flow topology is seen to persist. The resistivity allows reconnection to proceed, burning up magnetic flux and energy in the current sheet, resulting in Figs. 1(e) and 1(f) which show the field structure and flow topology at long times.

The current sheet at $x=x_{m}$ is not immediately apparent in Fig. 1(c). This is shown more clearly in the current density surface plot in Fig. 2(a) taken at the same time as Fig. 1(c). The current sheet appears as a large minimum in current density at the right-hand end. Looking from right to left along the axis we see a current maximum due to the original current channel. Also apparent is a current density maximum at the left-hand end. This is a second current sheet and is totally different in character to the primary sheet at $x=x_{m}$ in that material and magnetic flux are not

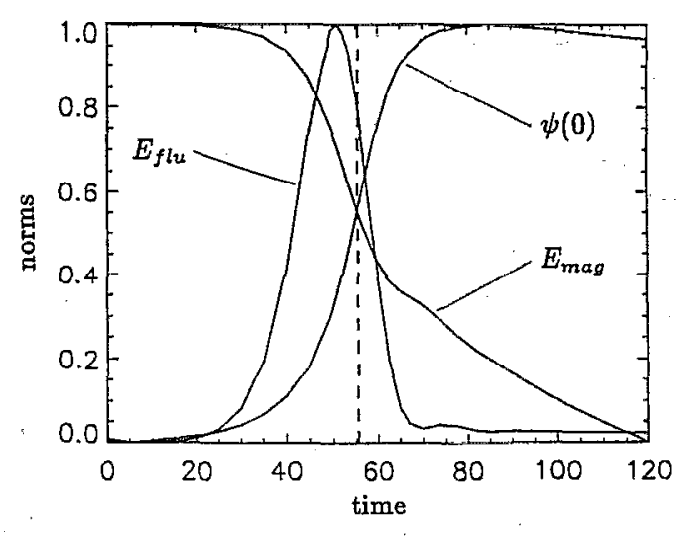

FIG. 3. Representative time histories of the total magnetic energy $\left(E_{\text {mag }}\right)$, the total fluid kinetic energy $\left(E_{\text {ffu }}\right)$, and the flux function at the $X$ point $[\psi(0)]$. The vertical dashed line marks the time of peak current in the current sheet.

being strongly driven into it, as can be seen in Fig. 1(d). Fast reconnection does not occur here. Indeed, this sheet persists long after the primary sheet has decayed. These secondary sheets have previously been noted by Richard et $a l^{22}$ in their study of the coalescence with regard to magnetic flux ropes. They refer to them as "anticoalescence" points. That this "anticoalescence" point is indeed a result of the linear instability can be most easily demonstrated by running the code with $\eta=0$, and using an artificial equation of motion to remove the fluid energy. This technique retains the exact form of the ideal instability, while damping the overshoots associated with the ideal system. The resultant current density surface is shown in Fig. 2(b). The presence of both current sheets is now very clear.

\section{B. Time development}

Representative time histories of the magnetic energy, the fluid kinetic energy, and the flux function at the $\mathrm{X}$ point are plotted in Fig. 3 for a purcly two-dimensional case with $\eta=5 \times 10^{-3}$. Each data set has been suitably normalized to fit the vertical axis range of $0-1$. The vertical dashed line marks the time of maximum current in the sheet. The initial kinetic energy is that due to the perturbation added at $t=0$. At early times the kinetic energy decays but after a few Alfvén times the unstable eigenfunction develops, resulting in the exponential rise in the kinetic energy. Note that the kinetic energy peaks before maximum current. This is a characteristic of all our coalescence simulations. At long times, the energies are slowly decaying as the new equilibrium is approached. The magnetic energy continues to decrease, even after the activity associated with the current sheet has ceased, as evidenced by the relatively benign changes in the flux function at long times. The resistive time scale for the $\eta$ values we use is still significant, hence the continued resistive decay of the magnetic energy.

To estimate the magnetic energy removed purely by the current sheet, we subtract the magnetic energy at the 
time that reconnection via the current sheet has ceased from the initial magnetic energy. The time at which the fast reconnective phase ends coincides closely with the change in slope of the magnetic energy-in this case, about 65 Alfven transit times. We therefore estimate the energy change to be 0.532 . This is the so-called "topological energy" which can only be removed via reconnection. To give some scale to this figure we need to relate it to a characteristic equilibrium magnetic energy. On examining the equilibrium fields, we find that $B_{x}$ behaves like $\tanh (y)$ at large $y$, and therefore represents an ever increasing sink of magnetic energy as $y_{m}$ is increased. However, a good approximation to the asymptotic field structure is obtained when $y \sim 2$ in our units. The equilibrium magnetic energy within $y=2$ is 4.708 for $\epsilon=0.3$, so that about $12 \%$ of that energy has been removed resistively.

\section{Scaling laws}

We now turn our attention to the scaling of the current sheet with resistivity. As mentioned previously, the sheet structure at the time of maximum current in the sheet as a function of $\eta$ is seen as a reliable indicator of fast reconnection. The linear theory suggests that any time up to maximum current will actually suffice. However, we find (consistent with previous studies) that the time at which maximum current is achieved actually increases with reductions in $\eta$. Therefore diagnosing the sheet at the time of maximum current has the advantage of being a simple, consistent datum point to identify. Using this diagnostic, then, our first result is as expected-the length $L$ of the

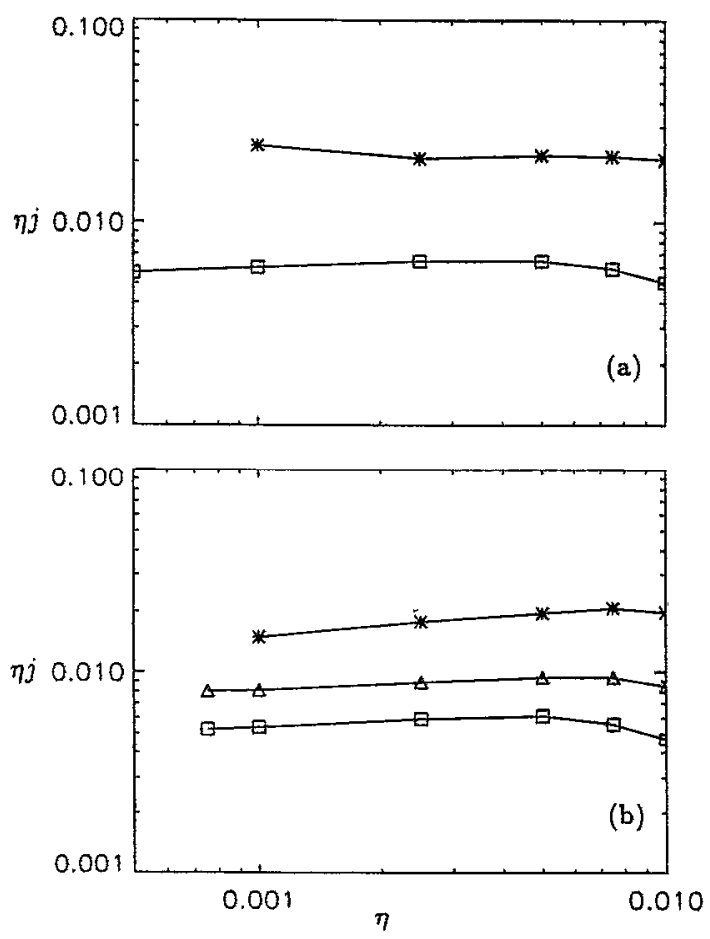

FIG. 4. Plots of $\eta j$ against $\eta$ for (a) $P_{0}=0.1$ (*) and 1 (a), and (b) $P_{0 z}=0.5(*), 1.0(\Delta)$, and $1.5(\square)$. current sheet is always approximately constant with $\eta$ (as in BW, DC, and Strauss). What determines $L$ is the amplitude of the external factors driving the system, at least within limits set by the global geometry.

A consequence of the BW scaling is that $\eta j-$ invariant. This dependence is plotted in Figs. 4(a) and 4 (b). The crosses mark the raw data from a given run, and each set of points for a given equilibrium are joined together. To a good approximation, the invariance of $\eta j$ is well confirmed in all situations. This result has also been confirmed by Richard et al. in their simulations. We note that a slight underachievement for the largest value of $\eta$ was discussed by DC. Effectively, the largest $\eta$ value is operational in decaying the field structure before the current sheet has formed, thereby underestimating the current sheet intensity. Even if the impact of the resistivity on the equilibrium fields is subtracted out of Eqs. (18a) and (18b) [as we do in our study, following BW with their inclusion of an " $E_{0}$ " in their Eq. (1)], the effect of the largest $\eta$ values is still felt. Whatever, the important point is that as we reduce $\eta$ we find no significant change in the invariant scaling of $\eta \dot{j}$.

Another sensitive indicator of the BW scaling is $W_{\eta}$ which is given by the integral of $\eta j^{2}$ over the current sheet. The $\eta j$ scaling is taken at the $\mathrm{X}$ point where the maximum current occurs. There is no ambiguity in its measurement. However, to find $W_{\eta}$ we need to make a distinction as to whether a given grid point lies inside or outside the current sheet. Thus, $W_{\eta}$ will be sensitive to how well we can resolve the current sheet, more so than the measurement of $\eta j$. We choose to define a grid point to be in the current
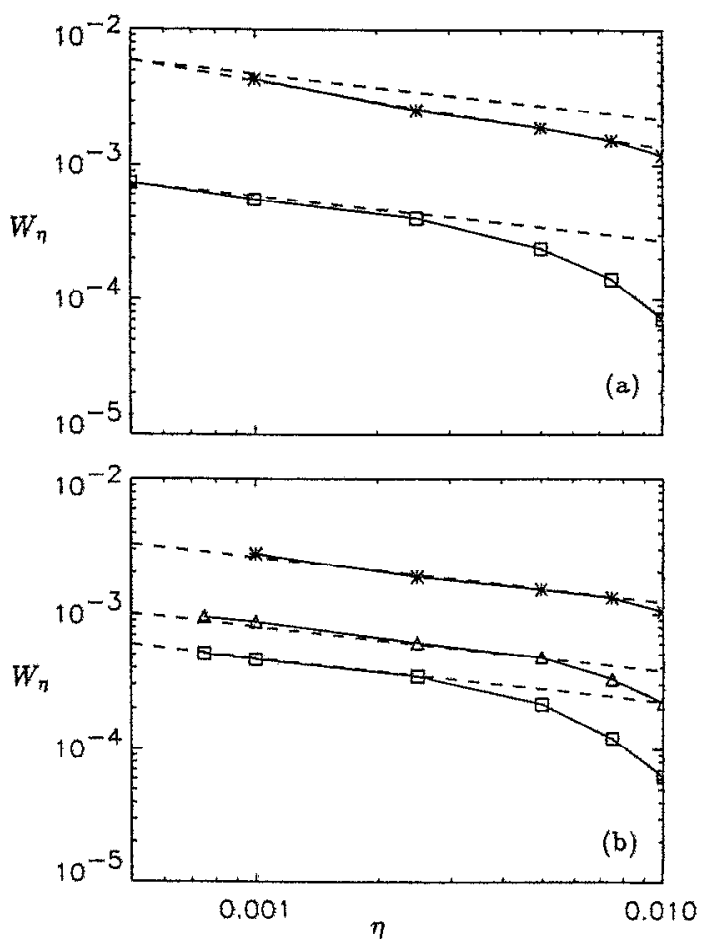

FIG. 5. Plots of $W_{\eta}$ against $\eta$ for (a) $P_{0}=0.1(*)$ and 1 ( $\square$ ), and (b) $P_{0 z}=0.5(*), 1.0(\Delta)$, and $1.5(\square)$. 

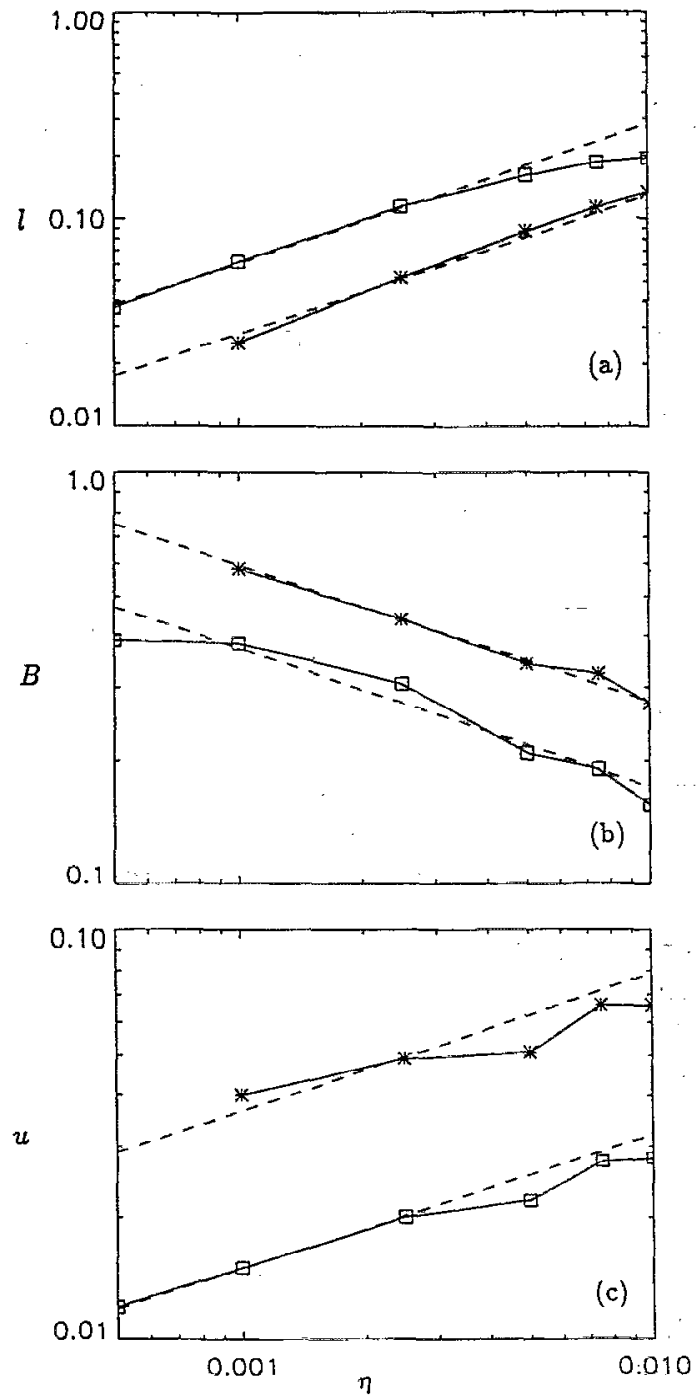

FIG. 6. Plots of (a) current sheet width $l$, (b) characteristic magnetic field at current sheet surface $B$, and (c) inflow speed $u$, for $P_{0}=0.1$ (*) and $1(\square)$.

sheet if its current is greater than or of the order of onethird of the current maximum. Figures 5(a) and 5(b) show the resultant dependence of $W_{\eta}$ on $\eta$. Given the modest resolution, the fit to an $\eta^{-1 / 3}$ scaling shown by the dashed lines is surprisingly good. In the run with $P_{0}=0.1$ in Fig. 5(a) we actually seem to overestimate the gradient over the range of $\eta$ used. Indeed a fit to a scaling of $\eta^{-1 / 2}$ is tantalizingly good.

Further confirmation of the BW scaling at peak current is shown in Figs. 6 and 7, where the sheet width, the magnetic field at the edge of the sheet, and the inflow velocity into the sheet are plotted as functions of $\eta$. Figure 6 is for the cases $P_{0}=0.1$ and 1, Fig. 7 is for the remainder. Again, the fit to the BW scalings highlighted by the dashed lines is remarkably good over the range of $\eta$ used. The sheet magnetic field and the inflow velocity are measured directly from the simulations. The sheet width is found by fitting the current profile across the sheet to
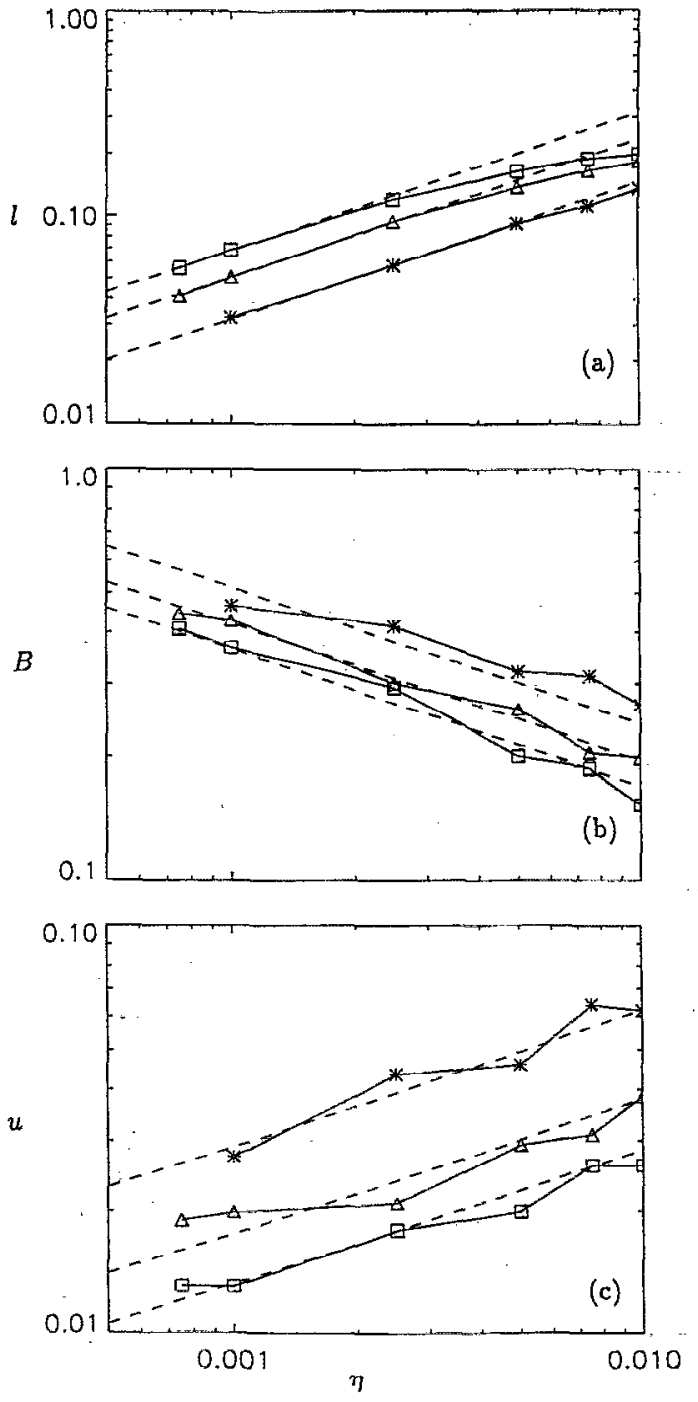

FIG. 7. Plots of (a) current sheet width $l$, (b) characteristic magnetic field at current sheet surface $B$, and (c) inflow speed $u$, for $P_{0 z}=0.5$ $(*), 1.0(\Delta)$, and $1.5(\square)$.

$J(x)=J_{\max } /\left[\cosh \left(x-x_{0} / l\right)\right]^{2}$, where $x_{0}$ is the current maximum location. This is the technique advocated by Biskamp, and subsequently used by DC. We also note that the $\eta$ range is reduced for the runs that are most compressible, i.e., $P_{0}=0.1$ and $P_{0 z}=0.5$. Again this is due to the severity of the scaling of the sheet width. Each set of simulations has been pushed as far as possible in $\eta$ without unduly compromising the resolution across the current sheet, and allowing the runs to be completed in a reasonable time.

Missing from Figs. 6 and 7 is the scaling for the outflow speed $v$ from the sheet. The incompressible scalings predict $v \sim \eta^{-1 / 3}$, i.e., the speed scales as the Alfvén speed at the sheet edge. Incompressibly, such a scaling is to be expected. However, results from our simulations show that $v$ is at most insensitive to $\eta$, and may actually be weakly decreasing with $\eta$. We attribute this to the extra degrees of freedom introduced by compressibility. We find that the 
disparity between the internal and external densities of the sheet, $\rho_{e}$ and $\rho_{i}$, becomes increasingly marked with reductions in $\eta$. Although the high-mass densities and pressures in the sheet do not build up sufficiently to stall the inflow, the (incompressible) scaling of the outflow is no longer followed.

Finally we mention that for sufficiently high aspect ratios the sheet is expected to become tearing mode unstable. In the present computations the aspect ratio remains well below 100-a typical level for the onset of the tearing mode according to Biskamp-and we see no evidence for the breakup of the current layer.

\section{DISCUSSION AND CONCLUSIONS}

We have confirmed the fast reconnective scalings observed by BW during the nonlinear development of the coalescence instability. These scalings are completely consistent with the theoretical arguments given in Sec. II which provide scaling laws for the sheet parameters with resistivity. The fast scalings are also retained in the case of compressible force-free equilibria which comprise a nonplanar component of the magnetic field. In all cases the compressibility of the plasma is reflected mainly in the outflow characteristics of the sheet. The exhaust flow is generally driven more weakly but all other scalings closely approximate the incompressible case.

Within the moderate compressibility range considered by us, we find that the plasma compressibility does not significantly alter the fast scalings, at least for $\epsilon=0.3$ equilibria. We have not, however, explored the time development in detail. Several workers have specifically investigated the role of compressibility on the coalescence (Bhattacharjee et al. and Tajima and Sakai), and have highlighted the appearance of time-dependent effects on the reconnection speed at fixed values of the resistivity. In many respects these investigations are complementary to our own. They show that the temporal behavior of $\epsilon=0.7$ equilibria is significantly different from those using $\epsilon=0.3$ in that an acceleration of the reconnection rate is observed (as also seen by Brunel et al. ${ }^{23}$ ).

A crucial question is whether the fast scaling persists at realistic values of plasma resistivity. In their discussion of the coalescence, BW argue that as $\eta$ continues to be reduced, the $W_{\eta}$ scaling must then saturate at some critical value of $\eta, \eta_{c}$ say. They suggest that the saturation occurs because the characteristic $B$ associated with the sheet will ultimately be limited to the value of the equilibrium magnetic field some distance from the island. They claim that the scaling reverts back to Sweet-Parker, so that the fast scaling observed at moderate $\eta$ values will turn over and become slow. Coincidentally, the kind of $\eta$ values at which they surmise this will occur is beyond the scope of our present simulations (typically $\eta_{c} \approx 2 \times 10^{-4}$ ) so we are unable to test their hypothesis numerically. However, if we assume that the reconnection stalls when the magnetic intensity at the sheet begins to exceed the level of the external field, then, by an extrapolation of our results, $\eta_{c} \approx 1 \times 10^{-4}$.
Either way, it is clear from our scaling analysis that a continual flux pileup at the onset of the current sheet is a necessary condition for fast reconnection. The same is true in the analysis of DC-and indeed for any quasi-onedimensional current sheet of fixed length. If the reconnection rate stalls as $\eta$ is reduced then the magnetic energy that emerges at the onset of the current layer can no longer be regarded as invariant. The absence of significant fiux pileup in this case means that the reconnection rate becomes slow-it reverts back to Sweet-Parker. Interestingly, DC also see a hint of a saturation in their scalings, again around the same level $\eta_{c}$. Whether this saturation is a physical charateristic of reconnection, or is merely an artifact of lack of resolution, has yet to be determined.

The flow topology appears to be a critical factor in determining the scaling of the reconnection. In particular, flow patterns that prevent the gas pressure from damping the buildup of current by allowing the circulation of material seem to play an important role. This is true here [see Fig. 1(d)] and also in DC. The DC simulations are in a doubly periodic domain so that circulation is ensured. In the coalescence there is a bounding wall, but the instability naturally forces the bulk of the flow to remain close to the separatrix, thereby neutralizing the impact of the wall. In the simple X-point geometry of Craig and Watson, however, the flow is unable to circulate and modest gas pressures (or finite perpendicular field components) can stall the reconnection. In this case the reconnection proceeds statically, i.e., at a rate directly proportional to the plasma resistivity.

${ }^{\prime}$ P. A. Sweet, Nuovo Cimento Suppl. 8, Ser. X, 188 (1958),

${ }^{2}$ E. N. Parker, Astrophys. J., Suppl., 77, 8 (1963).

${ }^{3} \mathrm{H}$. E. Petschek, in AAS/NASA Symposium on the Physics of Solar Flares, edited by W. N. Hess (National Aeronautic Space Administration, Washington, DC, 1964), p. 425.

${ }^{4}$ V. M. Vasyliunas, Rev. Geophys. Space Phys. 13, 303 (1975).

${ }^{5}$ T. G. Forbes and E. R. Priest, Rev, Geophys. 35, 1583 (1987).

${ }^{6}$ I. J. D. Craig and A. D. Sneyd, Astrophys. J. 357, 653 (1990).

${ }^{7}$ J. M. Finn and P. K. Kaw, Phys. Fluids 20, 72 (1977).

${ }^{8}$ P. L. Pritchett and C. C. Wu, Phys. Fluids 22, 2140 (1979).

${ }^{9} \mathrm{D}$. Biskamp and H. Welter, Phys. Rev, Lett. 44, 1069 (1980)

${ }^{10}$ E. E. Deluca and I. J. D. Craig, Astrophys. J. 390, 679 (1992).

"H. R. Strauss, J, Geophys. Res. 95, 17145 (1990).

${ }^{12}$ A. Bhattacharjee, F. Brunel, and T. Tajima, Phys. Fluids 26, 3332 (1983).

${ }^{13}$ T. Tajima and J. I. Sakai, Sov. J. Plasma Phys. 15, 606 (1989).

${ }^{14}$ D. Biskamp, Phys. Fluids 29, 1520 (1986).

${ }^{15}$ I. J. D. Craig and A. N. McClymont, Astrophys. J. Lett. 371, L41 (1991).

${ }^{16}$ I. J. D. Craig and A. N. McClymont, "Linear theory of fast reconnection at an X-type neutral point," Astrophys. J. (in press).

${ }^{17}$ I. J. D. Craig and P. W. Watson, Astrophys. J. 393, 385 (1992).

${ }^{18}$ V. M. Fadeev, I. F. Kuartskhava, and N. N. Komarov, Nucl. Fusion 5, $202(1965)$

${ }^{19}$ S. T. Zalesak, J. Comput. Phys. 31, 335 (1979).

${ }^{20}$ I. J. D. Craig and A. D. Sneyd, Comput. Math. Appl. 16, 341 (1988).

${ }^{21}$ I. J. D. Craig, A. N. McClymont, and A. D. Sneyd, Astrophys. J. 335, 441 (1988)

${ }^{22}$ R. L. Richard, R. J. Walker, R. D. Sydora, and M. Ashour-Abdalla, J. Geophys. Res, 94, 2471 (1989).

${ }^{23}$ F. Brunel, T. Tajima, and J. M. Dawson, Phys. Rev. Lett. 49, 323 (1982). 